\title{
Effect of dislocations on charge carrier mobility-lifetime product in synthetic single crystal diamond
}

\author{
A. Lohstroh, ${ }^{\text {a) }}$ P. J. Sellin, S. G. Wang, A. W. Davies, and J. Parkin \\ Department of Physics, University of Surrey, Guildford GU2 7XH, United Kingdom \\ R. W. Martin and P. R. Edwards \\ Department of Physics, SUPA, University of Strathclyde, John Anderson Building, 107 Rottenrow, Glasgow \\ G4 ONG, United Kingdom
}

(Received 29 May 2006; accepted 1 February 2007; published online 7 March 2007)

\begin{abstract}
The authors report correlations between variations in charge transport of electrons and holes in synthetic single crystal diamond and the presence of nitrogen impurities and dislocations. The spatial distribution of these defects was imaged using their characteristic luminescence emission and compared with maps of carrier drift length measured by ion beam induced charge imaging. The images indicate a reduction of electron and hole mobility-lifetime product due to nitrogen impurities and dislocations. Very good charge transport is achieved in selected regions where the dislocation density is minimal. @ 2007 American Institute of Physics. [DOI: 10.1063/1.2711754]
\end{abstract}

Diamond has been studied for many decades as a solid state ionization chamber for radiation detection. ${ }^{1,2}$ It combines a number of extreme properties, such as large charge carrier saturation velocities, radiation hardness, tissue equivalence, chemical inertness, and high thermal conductivity. Its large band gap of $5.48 \mathrm{eV}$ results in solar blindness and low leakage currents during room temperature operation, $^{3}$ for example, in high energy physics experiments, ${ }^{4}$ medical dosimetry, ${ }^{2}$ beam monitoring, ${ }^{4}$ and neutron detection. ${ }^{5}$

The detector performance and stability are determined by structural imperfections and impurities in the material, which influence the induced current signal. In polycrystalline diamond detectors it is known that grain boundaries act as charge trapping centers, severely limiting the spectroscopic performance. Ion beam induced charge (IBIC) imaging has been used routinely to study diamond radiation detectors, their priming behavior, and interactions due to optical illumination. ${ }^{6}$ Recently, single crystal chemical vapor deposited (CVD) diamond has been synthesized successfully in a number of laboratories. ${ }^{7-9}$ This material allows us to study the effect of defects on charge transport in the absence of grain boundaries.

In this work, we present a combination of luminescence and IBIC imaging in a synthetic single crystal diamond provided by Element Six Ltd. (UK). The spatial defect distribution of the material has been investigated using a photograph of the luminescence excited by $<227 \mathrm{~nm}$ wavelength ultraviolet (UV) irradiation of a xenon lamp. Cathodoluminescence (CL) spectral imaging at room temperature has been carried out at the University of Strathclyde ${ }^{10}$ with an electron energy of $10 \mathrm{keV}$ and typical beam currents of 4-10 nA. IBIC images were acquired at room temperature at the nuclear microbeam facility of the Surrey Ion Beam Centre. ${ }^{11}$ The IBIC spatial resolution is limited to $10 \mu \mathrm{m}$ by the number of pixels in the scan area.

Each proton interaction produces electron hole pairs, corresponding to a charge $Q_{0}$ in the device, and the charge carriers drift through the material according to the electric

\footnotetext{
${ }^{a)}$ Electronic mail: a.lohstroh@surrey.ac.uk
}

field. The induced current pulse is integrated by an Ortec 142 A charge sensitive preamplifier followed by an Ortec 570 shaping amplifier. The amplitude $Q$ of each interaction is proportional to the carrier drift length; it is calibrated in terms of charge collection efficiency $\mathrm{CCE}=Q / Q_{0}$, using a $100 \%$ efficient silicon pin diode. The average electron-hole pair creation energies are 3.6 and $13.2 \mathrm{eV}$ in silicon and diamond, respectively. ${ }^{12,13}$

The sample dimensions were $2.4 \times 2.9 \times 0.49 \mathrm{~mm}^{3}$, cut vertically through the wafer grown by a microwave enhanced plasma CVD method. During the growth process, nitrogen was deliberately introduced into the growth reactor for short periods of time, in order to study its effect on the material properties. Consequently, thin layers of nitrogen enriched material are found at various distances from the highpressure high-temperature (HPHT) diamond substrate. These layers are easily identified as the orange lines in the luminescence image shown in Fig. 1(a). Vertical line structures in blue are also distinguishable, associated with band $A$ luminescence from dislocation bundles in diamond. ${ }^{14}$ The CL confirms that outside the nitrogen doped areas only band $A$ luminescence is observed. While it is primarily concentrated in vertical dislocation bundles, it is also observed throughout the sample as low intensity pointlike luminescence centers, visible in Fig. 1(b). The HPHT synthesized substrate emits very bright blue luminescence and charged strongly during the electron irradiation of the CL experiment. The CL spectra shown in Fig. 1(c) are extracted from the indicated positions in Fig. 1(a). The spectrum extracted from the nitrogen doped layer exhibits the known signature of a neutral nitrogenvacancy complex ${ }^{15}$ and another nitrogen related defect signal with a peak position around $2.33 \mathrm{eV} .^{16}$

For the IBIC experiments, Ohmic contacts were formed on the front and on the back side of the sample using evaporated $\mathrm{Ti} / \mathrm{Au}(50 \mathrm{~nm} / 30 \mathrm{~nm})$, which were annealed at $673 \mathrm{~K}^{17}$ A $2.58 \mathrm{MeV}$ proton beam is electrostatically scanned over the investigated sample surface, with typically less than $1500 \mathrm{~s}^{-1}$ incident particles. The detector bias $V$ was connected to the irradiated contact via the preamplifier, whereas the back electrode was grounded. The range of the particles is $x=35 \mu \mathrm{m},{ }^{18}$ which is small compared to the 

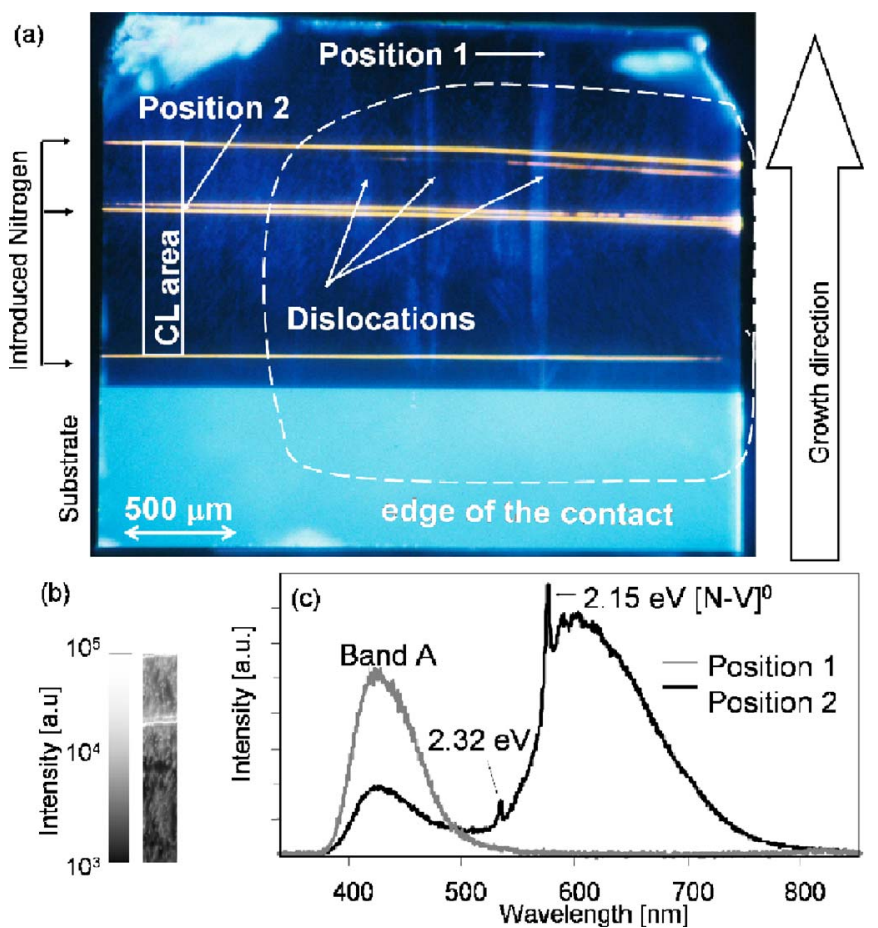

FIG. 1. (Color online) (a) Luminescence image (courtesy of Element Six Ltd., UK) taken prior to contact deposition. The outline of the subsequent position of the contact is indicated, as well as areas where CL measurements were taken. (b) Panchromatic CL image of the area indicated in (a). (c) CL spectra.

sample thickness $d=0.49 \mathrm{~mm}$. Consequently, pulses acquired under positive bias supply are sensitive to hole transport, whereas pulses acquired under negative bias are produced by electron drift.

Charge carrier mobility-lifetime products $(\mu \tau)$ can be measured using the modified Hecht equation for single charge carrier transport given in expression (1), which describes the CCE as a function of $V$ under the assumption of a constant electric field throughout the detector thickness $d .^{12}$ A constant offset $x / d$ has been added to account for the small contribution of the opposite charge carrier type,

$$
\mathrm{CCE}=\frac{\mu \tau V}{d^{2}}\left[1-\exp \left(-\left(1-\frac{x}{d}\right)\left(\frac{d^{2}}{\mu \tau V}\right)\right)\right]+\frac{x}{d} .
$$

IBIC maps of the detector response were taken at a number of bias voltages between 30 and $150 \mathrm{~V}$ for both polarities. Figure 2 displays a typical CCE map acquired at $V$ $\geqslant 100 \mathrm{~V}$ for both electron and hole signals. The top edge of

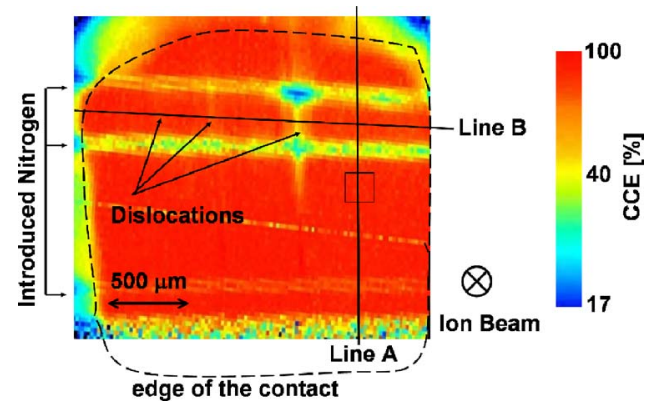

FIG. 2. (Color online) CCE map sensitive to hole transport, acquired at $+125 \mathrm{~V}$. The rectangular area has been used to extract the PHS spectra

shown by the solid lines in Fig. 3.
Downloaded 30 Mar 2009 to 131.227.178.132. Redistribution subject to AlP license or copyright; see http://apl.aip.org/apl/copyright.jsp

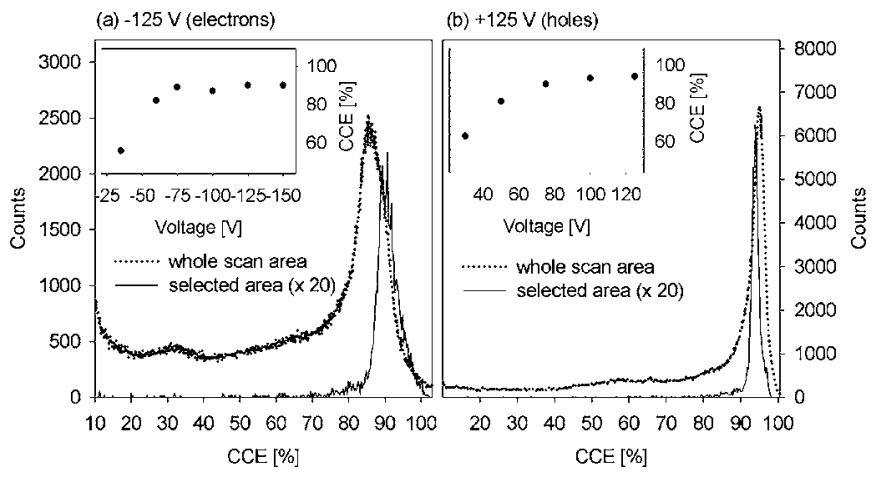

FIG. 3. CCE histograms of (a) electrons and (b) holes. The inset shows the CCE peak position of the histograms acquired over the selected area as a function of bias voltage.

the contact is not well defined in the IBIC data, which is probably caused by a small displacement and/or silver paint at the rear contact. The HPHT substrate does not show any induced signals above the threshold level of the system.

The overall pulse height spectra averaged over the total scan area shown in Fig. 3 exhibit well resolved peaks for electron and hole dominated signals. For comparison, pulse height spectra extracted from a rectangular selected regionindicated in Fig. 2-are also displayed, which demonstrate a reduction in peak width indicating the more homogeneous response in areas which are less affected by defects. In general, the width of the hole peak is narrower than for electrons and more than $95 \% \mathrm{CCE}$ has been reached, corresponding to a mean drift length $\mu \tau V / d$ of more than $3 \mathrm{~mm}$.

The insets in Fig. 3 show the CCE peak positions from the selected area as a function of bias supply. Polarization was observed during the irradiation of the sample, modifying the electric field experienced by the moving charges. The polarization field varied with sample position ${ }^{19}$ and introduces variations in the behavior expected by the Hecht equation [Eq. (1)]. $\mu \tau$ in the selected area is estimated as $6 \pm 2$ $\times 10^{-5} \mathrm{~cm}^{2} \mathrm{~s}^{-1}$ for electrons and $8 \pm 3 \times 10^{-5} \mathrm{~cm}^{2} \mathrm{~s}^{-1}$ for holes.

These values are two orders of magnitude below the values reported by Isberg et al. $^{20}$ which have been measured using higher created charge densities produced by UV irradiation. Published values for carrier mobilities in diamond vary from 1000 up to $4500 \mathrm{~cm}^{2} \mathrm{~V}^{-1} \mathrm{~s}^{-1}$. 3,7,21,22 Accordingly, the carrier lifetimes in our samples are in the order of a few tens of nanoseconds.

The reduced signal height along the nitrogen enriched lines is shown very clearly in Figs. 2 and 4(a). These data directly confirm that nitrogen impurities reduce the $\mu \tau$ of electrons and holes, consistent with a nitrogen related defect acting as a recombination center. ${ }^{23}$ Although less strong, the bands showing strong band $A$ luminescence intensity also exhibit degraded electron and hole charge transports compared to their surroundings, illustrated in Fig. 4(b). The areas of enhanced band $A$ luminescence emission, corresponding to dislocations, coincide clearly with reduced CCE; thus dislocations reduce $\mu \tau$ for both carrier types and the same line of argument as for the nitrogen lines also indicates a recombination center in this case. ${ }^{23}$ On the other hand, the "fading" of the dislocation effect visible in Fig. 2 towards the substrate area demonstrates that a homogeneous CCE profile can be achieved despite the presence of dislocations at high field strength, i.e., at high charge carrier velocities. The fields ap- 


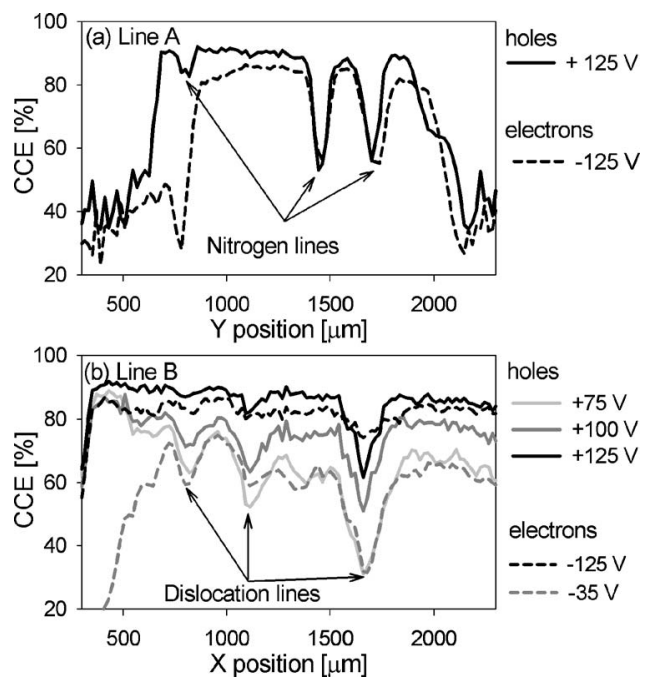

FIG. 4. CCE cross sections of various bias voltages. (a) extracted from line $\mathrm{A}$ and (b) extracted from line B indicated in Fig. 2.

plied in this study have a maximum strength of 3 $\times 10^{3} \mathrm{~V} \mathrm{~cm}^{-1}$, which is below the saturation regime of the carrier velocities. ${ }^{13}$ The weaker intensity band $A$ pointlike structures visible in the luminescence image are not resolved in the IBIC experiment, which could be either due to their weak effect on the charge transport or due to the limited spatial resolution.

In conclusion, it is found that dislocations strongly emitting band $A$ luminescence (compared to their surroundings) have reduced mobility-lifetime products of electrons and holes. A spatially homogeneous and high CCE profile can be achieved at field strength above $2 \times 10^{3} \mathrm{~V} \mathrm{~cm}^{-1}$ in selected areas over a millimeter length scale. Additionally, the study confirms the degradation of charge transport due to the presence of nitrogen in synthetic single crystal CVD diamond.

${ }^{1}$ A. Mainwood, Semicond. Sci. Technol. 15, R55 (2000).

${ }^{2}$ P. Bergonzo and R. B. Jackman, in Thin-Diamond II, Semiconductors and
Semimetals Vol. 77, edited by C. E. Nebel and J. Ristein (Elsevier, New York, 2004), Chap. 6, pp. 197-309.

${ }^{3}$ Properties, Growth and Applications of Diamond, Electronics Materials Information Service Vol. 26, edited by M. H. Nazaré and A. J. Neves, (Elsevier, New York, 2001), pp. 40-51.

${ }^{4}$ H. Kagan, Nucl. Instrum. Methods Phys. Res. A 541, 227 (2005).

${ }^{5}$ M. Pillon, M. Angelone, and A. V. Krasilnikov, Nucl. Instrum. Methods Phys. Res. B 101, 473 (1995).

${ }^{6}$ C. Manfredotti, Diamond Relat. Mater. 14, 531 (2005), and references therein.

${ }^{7}$ J. Isberg, J. Hammersberg, E. Johansson, T. Wikström, D. J. Twitchen, A. J. Whitehead, S. E. Coe, and G. A. Scarsbrook, Science 297, 1670 (2002).

${ }^{8}$ T. Teraji, S. Yoshizaki, S. Mitani, T. Watanabe, and T. Ito, J. Appl. Phys. 96, 7300 (2004).

${ }^{9}$ A. Tallaire, J. Achard, F. Silva, R. S. Sussmans, and A. Gicquel, Diamond Relat. Mater. 14 (2005).

${ }^{10}$ R. W. Martin, P. R. Edwards, K. P. O’Donnell, M. D. Dawson, C.-W. Jeon, G. R. Rice, and I. M. Watson, Phys. Status Solidi A 201, 665 (2004).

${ }^{11}$ A. Simon, C. Jeynes, R. P. Webb, R. Finnis, Z. Tabatabaian, P. J. Sellin, M. B. H. Breese, D. F. Fellows, R. van den Broek, and R. M. Gwilliam, Nucl. Instrum. Methods Phys. Res. B 219-220, 405 (2004).

${ }^{12}$ G. F. Knoll, Radiation Detection and Measurement, 3rd ed. (Wiley, New York, (1999), pp. 480-483.

${ }^{13}$ C. Canali, E. Gatti, F. Kozlov, F. Manfredi, C. Manfredotti, F. Nava, and A. Quirini, Nucl. Instrum. Methods 160, 73 (1979).

${ }^{14}$ J. Ruan, K. Kobashi, and W. J. Choyke, Appl. Phys. Lett. 60, 3138 (1992).

${ }^{15}$ K. Iakoubovskii, G. J. Adriaenssens, M. Nesladek, and L. Stals, Diamond Relat. Mater. 8, 717 (1999).

${ }^{16}$ N. C. Burton, J. W. Steeds, G. M. Meaden, Y. G. Shreter, and J. E. Butler, Diamond Relat. Mater. 4, 1222 (1995).

${ }^{17}$ T. Tachibana, B. E. Williams, and J. T. Glass, Phys. Rev. B 45, 11975 (1992).

${ }^{18}$ J. P. Biersack and L. Haggmark, Nucl. Instrum. Methods 174, 257 (1980).

${ }^{19}$ P. J. Sellin, A. Lohstroh, A. W. Davies, J. M. Parkin, M. Veale, M. B. H. Breese, and A. Simon, Nucl. Instrum. Methods Phys. Res. B (in press).

${ }^{20}$ J. Isberg, J. Hammersberg, H. Bernhoff, D. J. Twitchen, and A. J. Whitehead, Diamond Relat. Mater. 13, 872 (2004).

${ }^{21}$ M. Pomorski, E. Berdermann, M. Ciobanu, A. Martemyianov, P. Moritz, M. Rebisz, and B. Marczewska, Phys. Status Solidi A 202, 2199 (2005).

${ }^{22}$ H. Pernegger, S. Roe, P. Weilhammer, V. Eremin, H. Frais-Köbl, E. Griesmayer, H. Kagan, S. Schnetzer, R. Stone, W. Trischuk, D. Twitchen, and A. Whitehead, J. Appl. Phys. 97, 073704 (2005).

${ }^{23}$ L. S. Pan, D. R. Kania, P. Pianetta, J. W. Ager III, M. I. Landstrass, and S. Han, J. Appl. Phys. 73, 2888 (1993). 